\title{
Active Control Experiment Study of a Flexible Beam with Multiple Time Delays
}

\author{
Chen Long-Xiang and Cai Guo-Ping \\ Department of Engineering Mechanics, Shanghai Jiaotong University, Shanghai 200240, P.R. China
}

(Received 25 April 2013; accepted 3 March 2014)

In this paper, active control for vibration of a flexible beam with multiple time delays is studied numerically and experimentally. Piezoelectric (PZT) patches are used as actuators, and foil gauges were used as sensors. Firstly the motion equation of a flexible beam with multiple time delays and Piezoelectric patches is presented and written into a state space form. Then the state equation is discretized and transformed into a standard form without any explicit time delay by a particular augmentation for state variables. So time-delay controller could be designed based on the standard state equation using the discrete active control method. Finally, numerical and experimental studies are presented to verify the validity of the time-delay processing method using the discrete optimal control method and the discrete variable structure control method, respectively. An experimental setup is constructed using DSP TMS320F2812. The numerical and experimental results show that the proposed time-delay controller is effective in suppressing the beam vibration. It is also applicable to both short- and long- time delays.

\section{INTRODUCTION}

Time delay exists inevitably in active control systems. It may make the actuator input energy into the controlled systems when energy is not needed. This may cause the degradation of control efficiency or even the instability of control systems. ${ }^{1}$ Therefore, the research on time delay is of important theoretical significance and practical value.

Generally, the investigations on time delay may be divided into two classes: elimination and utilization technologies. At first, time delay was regarded as a "bad" factor that had only negative side effect on control systems. In order to eliminate or weaken the effect of time delay, some methods were subsequently proposed, including Taylor series expansion, phase shift technique and state pre-estimation. ${ }^{2-4}$ These methods can deal effectively with some short time delay problems in control systems, but awkwardly with long ones. Cai and Huang have proposed a new time-delay controller., ${ }^{5,6}$ This controller is designed directly from time-delay differential equation without any hypothesis in whole process of controller design, applicable for both short and long time delays. Chen proposed a theoretical method for a flexible beam with multiple time delays using the discrete optimal control. ${ }^{7}$ Sun has recently proposed a continuous time approximation method for linear and nonlinear dynamical systems with time delay. ${ }^{8}$ The key point of the methods mentioned above to eliminate the negative effect of time delay is so-called time-delay elimination technology or time-delay compensation technology. Its main function is to eliminate or weaken the negative effect of time delay on control efficiency. On the other hands, recent investigations have shown that voluntary introduction of delay into control systems can also benefit the control. For example, in nonlinear dynamics area, achievement is remarkable using time delay to control chaos motion. ${ }^{9}$ Daqaq, Alhazza and Arafat studied the effect of feedback delays on the non-linear vibration of a cantilever beam. ${ }^{10}$ In structural control area, Hosek and Olgac developed a time-delay resonator that may be used for vibration control of structures. ${ }^{11}$ Cavdaroglu and Olgac considered the cart-and-pendulum system as research object, this study shows that systems with multiple delays may exhibit better performance by increasing the delays to more desirable levels. ${ }^{12}$ Liu, Haraguchi, and Hu presents a reduction-based linear quadratic control for the dynamic system with a constant or a slowly time-varying input delay. ${ }^{13}$ In robotics area, Cai and Lim designed a time-delay controller for a flexible manipulator and their results show that delayed feedback control design may possibly achieve much better control efficiency than the no-delay control design. ${ }^{14}$ In control system of pipeline transport, time delay may be utilized to enhance steady critical speed of flowing liquid. ${ }^{15}$ Time delay may be also used to improve system stability. ${ }^{16,17}$ Those researches above involving the active utilization of time delay is so-called time-delay utilization technology, which assumes time delay as a design parameter to obtain good control performance. Although up to now researches have been done much on the elimination and utilization of time delay, most of work is theoretical one but few on experiment.

In this paper, Piezoelectric (PZT) patches are used as actuators, foil gauges as sensors, active control for vibration of a flexible beam with multiple time delays is studied numerically and experimentally. The controller with time delays is designed using the discrete optimal control method and the discrete variable structure control method, respectively. The feasibility and efficiency of the time-delay controller are verified theoretically and experimentally. This paper is organized 